Original Paper $\quad$ http://ajol.info/index.php/ijbcs $\quad$ http://indexmedicus.afro.who.int

\title{
Étude ethnobotanique des plantes alimentaires utilisées en médecine traditionnelle dans la région Maritime du Togo
}

\author{
Stéphane EFFOE ${ }^{1 *}$, Efui Holaly GBEKLEY ${ }^{1,2}$, Mamatchi MÉLILA², \\ Amégninou $\mathrm{ABAN}^{1}$, Tchadjobo TCHACONDO ${ }^{1}$, Elolo OSSEYI ${ }^{1,3}$, \\ Damintoti Simplice KAROU ${ }^{2}$ et Kouami KOKOU ${ }^{4}$
}

\author{
${ }^{1}$ Laboratoire des Sciences Biomédicales, Alimentaires et de Santé Environnementale (LaSBASE), Université de \\ Lomé, Togo. \\ ${ }^{2}$ Laboratoire de Microbiologie et de Contrôle de Qualité des Denrées Alimentaires (LAMICODA), Université \\ de Lomé, Togo. \\ ${ }^{3}$ Laboratoire de Génie des Procédés et des Ressources Naturelles (LAGEPREN), Université de Lomé, Togo. \\ ${ }^{4}$ Laboratoire de Recherche Forestière (LRF), Université de Lomé, Togo. \\ *Auteur correspondant ; E-mail : stephaneeffoe@gmail.com
}

\section{RESUME}

Les plantes alimentaires contiennent des principes actifs doués de diverses propriétés médicinales pouvant intervenir dans le traitement de nombreuses maladies. Cette étude est consacrée au recensement des plantes ayant des potentiels nutritionnel et thérapeutique dans la région Maritime du Togo, dans le but de la valorisation de ces plantes. De juin à septembre 2017, une enquête ethnobotanique, basée sur l'utilisation des interviews individuelles à l'aide d'un questionnaire semi-structuré, a été réalisée auprès de 101 Praticiens de la Médecine Traditionnelle. Au total 86 espèces végétales appartenant à 72 genres et 36 familles ont été identifiées. Les Fabaceae et les Solanaceae (7 espèces chacune) ont été les plus représentées. Les espèces les plus citées ont été Ocimum gratissimum L. (10,48\%), Vernonia amygdalina Delile (6,71\%), Lactuca taraxacifolia (Willd.) Schum. (6,08\%) et Heliotropium indicum L. (5,66\%). Les feuilles $(77,85 \%)$, les fruits $(5,63 \%)$ et les racines $(4,26 \%)$ sont les organes les plus utilisées sur 799 recettes inventoriées. La principale forme galénique reste la sauce $(51,19 \%)$ et le mode principal d'administration est la voie orale $(90,74 \%)$. Concernant les maladies traitées, les affections du tube digestif sont au premier rang (43,80\%) suivies par des affections cardiovasculaires $(13,52 \%)$. Cette étude fournie une base de données sur des plantes ayant des potentiels nutritionnel et thérapeutique au Togo.

(C) 2020 International Formulae Group. All rights reserved.

Mots clés : Alicaments, potentiels nutritionnel et thérapeutique, sécurité alimentaire, Togo.

\section{Ethnobotanical study of some food plants used in traditional medicine in the Maritime region of Togo}

\begin{abstract}
Food plants contain active substances with various medicinal properties that can be used to treat many diseases. This study is devoted to the inventory of plants with nutritional and therapeutic potential in Maritime region of Togo, with the aim of promoting these plants. From June to September 2017, an ethnobotanical survey
\end{abstract}


was conducted among 101 Traditional Medicine Practitioners through individual interviews using a semistructured questionnaire. A total of 86 plants species belonging to 72 genera and 36 families were identified. Fabaceae and Solanaceae (7 species each) were the most represented. The most cited species were Ocimum gratissimum L. (10.48\%), Vernonia amygdalina Delile (6.71\%), Lactuca taraxacifolia (Willd.) Schum. (6.08\%) and Heliotropium indicum L. (5.66\%). The leaves $(77.85 \%)$, fruits $(5.63 \%)$ and roots $(4.26 \%)$ were the most plant parts used_out of 799 inventoried recipes. The main dosage form remains the sauce $(51.19 \%)$ and the main mode of administration is the oral route $(90.74 \%)$. Regarding the treated diseases, the digestive disorders are in first place $(43.80 \%)$, followed by cardiovascular diseases (13.52\%). This study provides a database of plants with nutritional and therapeutic potential in Togo.

(C) 2020 International Formulae Group. All rights reserved.

Keywords: Food plants, nutritional and therapeutic potentials, food security, Togo.

\section{INTRODUCTION}

Les plantes constituent des ressources végétales naturelles de proximité, essentielles à l'homme et pour la conservation de la biodiversité (OMS, 2012). Hormis les plantes cultivées, plusieurs milliers de plantes sauvages peu connues revêtent une grande importance socioculturelle et socioéconomique, et sont douées de propriétés nutritionnelles et thérapeutiques (Benem et Sanou-Nana, 2009 ; Benkhnigue et al., 2010). Les conditions du développement démographique de certains groupes humains, la structure même de leur civilisation, leur degré d'évolution, dépendent dans une large mesure des ressources végétales (Salhi et al., 2010). Les plus bénéfiques potentialités de ces plantes sauvages sont leurs propriétés nutritionnelles et surtout thérapeutiques. En Afrique, les plantes médicinales constituent des ressources précieuses pour la grande majorité des populations rurales dont plus de $80 \%$ s'en servent pour assurer leurs soins de santé (Jiofack et al., 2010). Leur plus-value est reconnue dans leur utilisation pour le traitement efficace de diverses affections chroniques, invalidantes ou incurables (OMS, 2012). Elles constituent une alternative idéale aux médicaments dits conventionnels dont les coûts sont relativement assez élevés pour les populations surtout rurales des pays en développement. L'intérêt que revêt l'utilisation des espèces végétales pour les populations s'explique dans le fait que ces plantes qui sont douées de propriétés thérapeutiques sont bien avant tout, ou pour la majorité, des plantes alimentaires (Dansi et al., 2008). Ce potentiel des plantes alimentaires est d'une très grande importance chez les populations rurales en Afrique et surtout pour les populations urbaines condamnées à l'alimentation industrielle. Elles constituent une ressource efficace dans la gestion du double fardeau nutritionnel dont souffre nombre de pays d'Afrique subsaharienne, marqués par l'avancée galopante de la malnutrition et des maladies métaboliques. Elles sont souvent consommées comme amuse-gueule, légumes, épices et condiments (Betti et Mebere-Yemefa, 2011) et constituent un moyen efficace de lutte contre la pauvreté (Betti et al., 2016).

De façon controversée, si les plantes sont indispensables à l'alimentation et aux soins de l'homme, plusieurs d'entre elles considérées comme sauvages parce que moins cultivées, sont menacées de raréfaction ou de disparition $\mathrm{du}$ fait de la dépréciation alimentaire ou aux actions anthropiques (exploitation forestière, expansion agricole, urbanisation). Les forêts en général et même les forêts sacrées, subissent des dégradations rapides et massives, entraînant la réduction de leur superficie, voire leur disparition complète (Kokou et al., 2005). La disparition de ces espaces, seuls témoins de l'élément forestier, affecte notamment les espèces végétales non ligneuses qu'ils abritent. En effet, ces espèces sont mal connues par une majeure partie de la population ; d'où leur négligence ou leur sousexploitation. Cette double réalité n'épargne pas le Togo qui est un pays à faible couvert végétal. Dans certaines de ses régions notamment, dans la région maritime, les exploitations agricoles s'alignent à perte de vue au détriment des 
espèces végétales pourvues de potentiels nutritionnel et thérapeutique. Avec l'appui des scientifiques, des recherches ont été effectuées sur les ressources végétales sauvages. Certains ont porté sur la menace de disparition des plantes alimentaires due à des facteurs écologiques et sociales (Akpavi, 2010) ou à l'exclusion des ressources végétales locales en faveur de l'adoption de régimes alimentaires dits évolués (Akpavi et al., 2011). D'autres travaux ont été effectués sur leurs propriétés biologiques telles que les activités antimicrobienne (Agban et al., 2013), antiplasmodiale (Agbodeka et al., 2016) et antidiabétique (Gbekley et al., 2015). Cependant, peu de travaux ont été effectués sur le double intérêt nutritionnel et thérapeutique des plantes alimentaires. La présente étude investiguant le patrimoine ethno-médicinal et ethno-nutritionnel serait alors une première dans la région maritime. Elle se propose de faire une investigation sur les plantes alimentaires et médicinales et de confirmer le double potentiel nutritionnel et thérapeutique des plantes afin de contribuer au renforcement des bases de données scientifiques.

\section{MATERIEL ET METHODES}

\section{Cadre géographique et population de l'étude}

La présente étude a été réalisée dans la région maritime du Togo (Figure 1) qui s'étend sur une superficie de $6100 \mathrm{~km}^{2}$, entre $1^{\circ} 20^{\prime}$ de longitude Ouest et $1^{\circ} 50^{\prime}$ de longitude Est, puis entre $6^{\circ} 10^{\prime}$ de latitude Sud et $6^{\circ} 60^{\prime}$ de latitude Nord, soit environ $10,78 \%$ de la superficie totale du Togo. Cette région est limitée au nord par la région des Plateaux, à l'ouest par la république du Ghana, à l'est par la république du Bénin et au sud, par l'Océan Atlantique. Elle est caractérisée par un climat de type subéquatorial avec une longue saison des pluies de mars à juillet (maximum de $1200 \mathrm{~mm}$ en juin) et une courte saison des pluies de septembre à novembre (maximum de $1000 \mathrm{~mm}$ en octobre). Les précipitations minimales pour les deux saisons sont respectivement de 184,4 $\mathrm{mm}$ et $6,9 \mathrm{~mm}$. La température annuelle moyenne est d'environ $27,5^{\circ} \mathrm{C}$ avec un maximum autour de $35,1^{\circ} \mathrm{C}$ pendant la saison sèche. La végétation de la région est composée des forêts disparates, des reliques de forêts galeries, savanes, prairies, fourrés littoraux ou marécageux halophiles. Les données démographiques de la région ont révélé une densité de 100 à 200 habitants par $\mathrm{km}^{2}$ pour les zones de fortes densités, et de 50 à 100 habitants par $\mathrm{km}^{2}$ pour les zones de plus faibles densités (Direction Générale de la Statistique et de la Comptabilité Nationale 2010). Les principaux groupes ethniques sont les Ewé, les Ouatchi, les Mina et les Guin (Gayibor, 2006 ).

\section{Méthodologie de l'enquête ethnobotanique Matériel technique}

Le matériel technique utilisé pour les investigations ethnobotaniques est composé d'une fiche d'enquête, d'un appareil de localisation GPS (marque Garmin), d'un appareil photo (marque Rollei), d'un sécateur, d'un outil constitué de carton et de vieux papiers journaux pour la conservation des spécimens.

\section{Technique d'échantillonnage}

La présente étude ethnobotanique est de catégorie descriptive. La technique d'échantillonnage non probabilistique ou non aléatoire par commodité a été utilisée. Elle s'est inspirée des techniques d'échantillonnage connues en ethnobotanique quantitative (Houéhanou et al., 2016).

\section{Choix des enquêtés}

L'enquête ethnobotanique a été réalisée de juin à septembre 2017, auprès des Praticiens de la Médecine Traditionnelle (PMTs) de 10 localités de la région Maritime du Togo. Elle a été effectuée à l'aide d'une fiche d'enquête chez deux catégories de PMTs, d'une part les phytothérapeutes et d'autre part, les herboristes parmi lesquels les ménages et les herboristes de marché. L'âge de 18 ans et plus ainsi qu'une bonne connaissance des potentiels nutritionnel et thérapeutique des plantes, a constitué un critère d'inclusion. $\mathrm{Ne}$ pas connaître les potentiels nutritionnel et thérapeutique des plantes et ne pas avoir 18 ans au moins, a constitué un critère d'exclusion. Un premier entretien a été effectué avec les PMTs pour leur 
donner une explication succincte des objectifs de l'étude et de l'importance des renseignements qu'ils allaient fournir, afin d'obtenir leur consentement à participer à l'étude.

\section{Collecte des données et identifications botaniques}

La collecte des données a été réalisée grâce à des interviews individuelles suivant un questionnaire semi-structuré rédigé pour la circonstance (Dansi et al., 2008). Le questionnaire a été axé sur sept (7) principaux points suivants : (i) l'identité de l'enquêté (nom, prénoms, âge et sexe) ; (ii) l'origine du savoir (initiation au sein de la famille ou dans un autre cadre) ; (iii) le statut du PMT ( PMT à temps plein ou à temps partiel); (iv) niveau d'instruction; (v) nom de la plante alimentaire utilisée en langue locale ou autres langues et les organes utilisées ; (vi) indications thérapeutiques de la plante ou noms des maladies traitées par la plante en la langue locale ou autres langues, les symptômes qui aident à poser le diagnostic ; (vii) le mode de préparation de recettes et leurs administrations. Après les interviews avec les PMTs, les échantillons de plantes ont été récoltés entre novembre 2017 et janvier 2018 et des photographies ont été prises sur les sites pour aider à l'identification des plantes. L'identification des plantes a été effectuée grâce à l'expertise des botanistes du Laboratoire de Biologie et d'Ecologie Végétale de la Faculté des Sciences de l'Université de Lomé par comparaison avec les spécimens de l'herbier de ladite Faculté des Sciences de l'Université de Lomé. Ensuite, les souches taxonomiques des bases de données en ligne «Ressources Végétales de l'Afrique Tropicale» (PROTA) sur les sites : www.prota.org et «International Plant Name Index » (IPNI) http://www.ipni.org, ont été utilisées pour confirmation.

\section{Traitement des données}

Pour évaluer et s'acquérir de l'importance socioculturelle des plantes aux potentiels nutritionnel et thérapeutique inventoriées, les données qualitatives de l'enquête ont été traduites en données quantitatives lors de la saisie dans le logiciel tableur Excel 2010. Les diverses variables traitées sont : les plantes citées, les recettes, les maladies traitées, les organes de plantes utilisés, les modes de préparation et d'administration. Trois (3) indices ethnobotaniques ont été évaluées : il s'agit de la Fréquence de citation FC, de la Contribution de chaque espèce aux recettes CPR (Dassou et al., 2014), et de l'Indice de confirmation ou Consensus d'informateurs ICF (Ilumbe et al., 2014 ).

Fréquence de citation $\boldsymbol{F C}$ : La fréquence de citation (FC) de chaque espèce a été évaluée pour apprécier la régularité dans la distribution de l'espèce végétale à l'aide de la formule 1 .

$F C=\frac{\mathrm{CP}}{\mathrm{CT}} \times 100 \quad$ (Formule 1)

Dans la formule 1, CP représente le nombre de fois où l'espèce est citée; tandis que CT représente le nombre total de citations.

Contribution des plantes aux recettes

$\boldsymbol{C P R}$ : La contribution de chaque plante dans la constitution des recettes (CPR) a été déterminée pour apprécier la fréquence d'implication de la plante dans les recettes à l'aide de la formule 2 .

$C P R=\frac{\mathrm{RP}}{\mathrm{RT}} \mathrm{x} 100 \quad$ (Formule 2)

Dans la formule 2, RP désigne le nombre de recettes sollicitant la plante et RT, le nombre total de recettes.

Indice de confirmation ou Consensus d'informateurs (ICF) : Le facteur (degré) de consensus d'utilisation ou Informant Consensus Factor (ICF) a été calculé pour apprécier les accords des informateurs sur l'utilisation des plantes alimentaires à usages thérapeutiques.

$I C F=\frac{\text { IP }}{\text { IT }} \quad$ Formule 3)

Dans la formule 3, IP est le nombre d'informateurs ayant cité une espèce ; alors que IT est le nombre total d'informateurs. L'ICF varie entre [0 - 1]. Une valeur faible, proche de 0 , indique que les informateurs sont en désaccord sur les usages proposés. Quand cette valeur est proche de 1, elle indique un accord élevé autour de l'utilisation de la plante. 


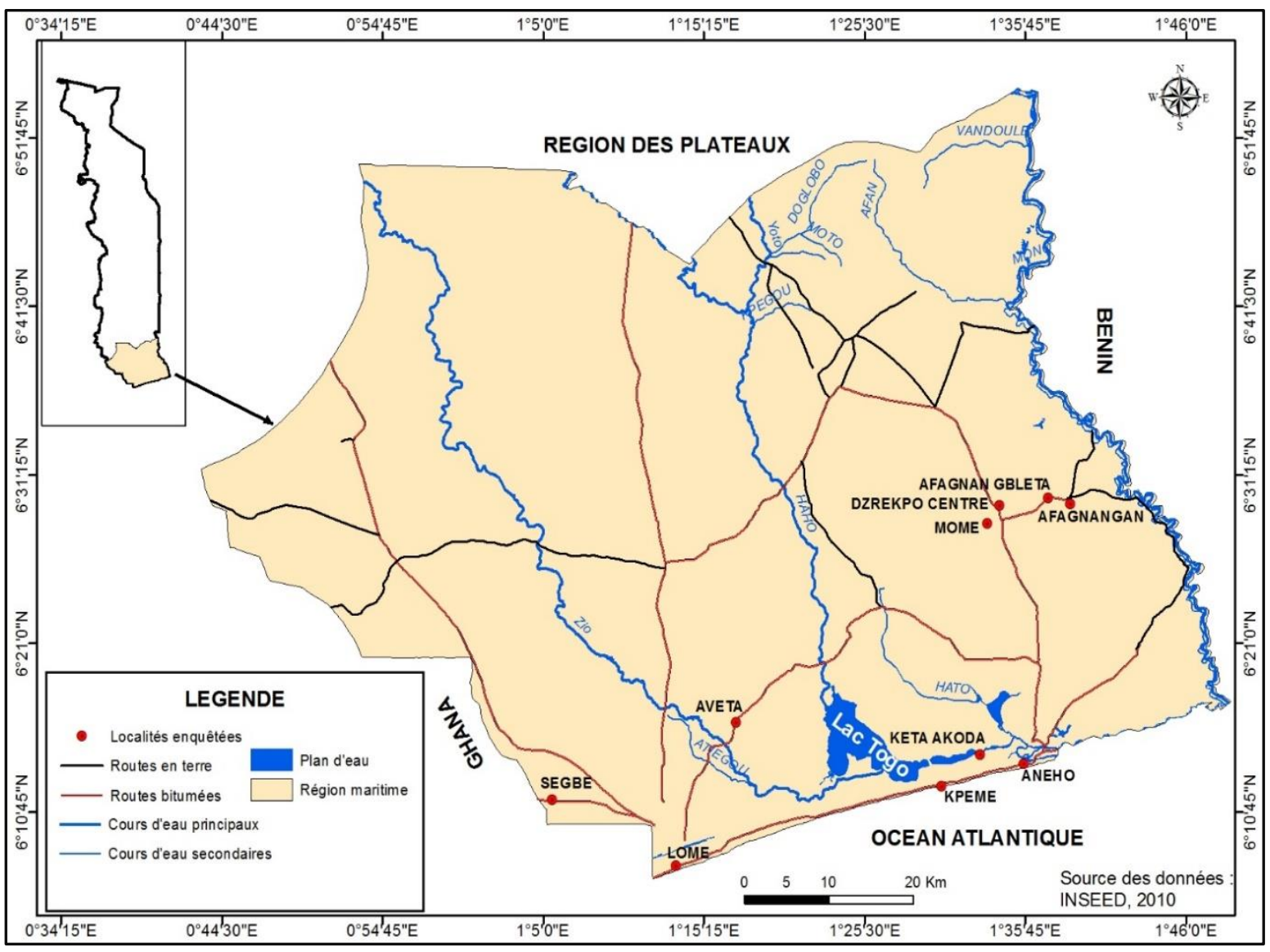

Figure 1 : Carte géographique de la région Maritime du Togo.

Source : Institut National de la Statistique et des Etudes Economiques et Démographiques. (INSEED) (Direction Générale de la Statistique et de la Comptabilité Nationale 2010).

\section{RESULTATS}

\section{Données sociodémographiques des PMTs}

La présente étude a enrôlé 101 PMTs de la région maritime du Togo dont 42 de sexe masculin et 59 de sexe féminin. Leur âge a varié de 18 à 100 ans avec une moyenne de 53,76 ans. Le Tableau 1 résume les données sociodémographiques de ces PMTs. Les PMTs ont été répartis en 5 classes d'âge d'amplitude égale à 20 ans. L'analyse des données sociodémographiques (Tableau 1) montre que la majorité des PMTs se retrouvent dans deux classes d'âge, soit de 40 à 60 ans et de 60 à 80 ans. Ces deux classes d'âge regroupent à elles seules $74,25 \%$ des enquêtés. Environ $61,39 \%$ de ces PMTs sont scolarisés; $35,64 \%$ ont atteint le niveau secondaire et $3,96 \%$ le niveau universitaire. Pour ce qui est de l'origine de leur savoir, la majorité, soit 74,26\%, a été initié à la pratique de la médecine traditionnelle au sein de la famille; tandis que les autres ont été initiés en dehors du cadre familial. Des PMTs enquêtés, les «Ouatchi » et les «Ewé » sont les plus majoritaires avec respectivement $61,39 \%$ et $13,86 \%$ d'enquêtés suivis des «Guin» $(11,88 \%)$ et des «Mina » $(6,93 \%)$. Les résultats de l'enquête ont aussi révélé que $32,67 \%$ des PMTs exercent la médecine traditionnelle en plein temps, tandis que les autres la pratiquent en activité secondaire.

\section{Diversité des plantes d'intérêts nutritionnel et thérapeutique}

$\mathrm{Au}$ total, 86 espèces végétales appartenant à 72 genres et 36 familles ont été 
répertoriées au cours de la présente étude comme intervenant dans 799 recettes. Les familles les plus représentées ont été les Solanaceae (7 espèces soit 8,14\%) : Capsicum annuиm L., Capsicum frutescens L., Physalis angulata L., Solanum aethiopicum L., Solanum americanum L., Solanum lycopersicum L., Solanum macrocarpum L., et les Fabaceae (7 espèces soit 8,14\%) : Cajanus cajan L., Cassia occidentalis L., Ceasalpinia pulcherrima L., Parkia biglobosa Benth., Pterocarpus santalinoides DC., Tamarindus indica L., Tetrapleura tetraptera (Schum. \& Thonn.). Les Amaranthaceae ont été représentés par 6 espèces soit 6,98\% suivies des Malvaceae avec 5 espèces soit $5,81 \%$, les Euphorbiaceae, les Annonaceae et les Asteraceae par 4 espèces soit $4,65 \%$ chacune (Figure 2). Au niveau des espèces végétales, $61(70,93 \%)$ ont été citées comme légumes parmi lesquelles 49 (56,98\%) comme légumes-feuilles (Figure 3).

\section{Saison et moment de récolte des espèces végétales}

Pour ce qui est de la saison et le moment de la récolte des organes de plantes, le facteur à considérer est la disponibilité des plantes pour les plantes saisonnières. Les plantes sont cueillies en toutes périodes de la journée et toutes saisons, vu que ce sont des plantes alimentaires.

\section{Importance des espèces végétales suivant les indices ethnobotaniques}

Les différents indices obtenus pour les 86 espèces ont été enregistrés dans le Tableau 2.

Selon la fréquence de citation (FC), les espèces les plus citées ont été $O$. gratissimum $(10,48 \%), \quad V . \quad$ amygdalina $(6,71 \%), \quad L$. taraxacifolia $(6,08 \%), H$. indicum $(5,66 \%)$, Moringa oleifera L. (5,45\%), Manihot esculenta Crantz (3,56\%), Corchorus aestuans L. $(2,94 \%)$ et Laportea aestuans L. (2,94\%).

Selon la contribution de chaque espèce aux recettes (CPR), les espèces les plus impliquées dans les 799 recettes ont été $O$. gratissimum (94 recettes ou 11,76\%), $V$. amygdalina (62 recettes ou 7,76\%), $L$. taraxacifolia (61 recettes ou 7,63\%), $H$. indicum (54 recettes ou 6,76\%), M. oleifera (53 recettes ou 6,66\%), M. esculenta (25 recettes ou 3,13\%), C. aestuans (21 recettes ou 2,63\%), Fagara zanthoxyloides Lam. (21 recettes ou 2,63\%) et Crataeva adansonii DC. (20 recettes ou $2,50 \%$ ).

Les indices de confirmation ou les Consensus d'informateurs (ICF) calculés sont inférieurs à 0,5 pour la plupart des espèces. La valeur moyenne de consensus $(0,5)$ a été obtenue pour $O$. gratissimum, suivie de $V$. amygdalina $(0,32), L$ taraxacifolia $(0,29), H$. indicum $(0,27)$ et $M$. oleifera $(0,26)$.

Utilisation des plantes selon la partie utilisée, la forme galénique et le mode d'administration

Plusieurs organes de plantes entrent dans la préparation des recettes. Il s'agit essentiellement des feuilles $(77,85 \%)$ suivies des fruits $(5,63 \%)$, des racines $(4,26 \%)$ et des autres parties présentées dans le Tableau 3. En ce qui concerne les formes galéniques, 51,19\% des recettes sont des recettes culinaires (sauces). Elles sont suivies par les jus $(14,52 \%)$, les décoctés $(14,14 \%)$ et les matières crues $(6,63 \%)$. Le principal mode d'administration est la voie orale $(90,74 \%)$ suivie de la voie cutanée $(3,00 \%)$ (Tableau 3 ).

\section{Utilisation des plantes selon les maladies traitées}

Au total 78 maladies regroupées en 17 catégories d'affections ont été répertoriées. Suivant leur fréquence de citation, les affections du tube digestif $(43,80 \%)$ sont les plus traitées par les plantes alimentaires recensées, suivies des affections cardiovasculaires $(13,52 \%)$ et des affections asthéniques (7,38\%) (Tableau 4). 
Tableau 1: Données sociodémographiques des PMTs de la région Maritime du Togo.

\begin{tabular}{|c|c|c|c|c|c|c|}
\hline \multirow[t]{2}{*}{ Sexes des PMTs } & Féminin & Masculin & & & & \multirow{2}{*}{$\begin{array}{l}\text { Total } \\
101 \\
(100,00 \%)\end{array}$} \\
\hline & $59(58,42 \%)$ & $42(41,58 \%)$ & & & & \\
\hline \multirow[t]{2}{*}{ Ages des PMTs } & $\leq 20$ ans & ]20-40] & ]40-60] & ]60-80] & ]80-100] & \multirow{2}{*}{$\begin{array}{l}\text { Total } \\
101 \\
(100,00 \%)\end{array}$} \\
\hline & $3(2,97 \%)$ & $18(17,82 \%)$ & $42(41,58 \%)$ & $33(32,67 \%)$ & $5(4,95 \%)$ & \\
\hline \multirow{3}{*}{$\begin{array}{l}\text { Niveau } \\
\text { d'éducation des } \\
\text { PMTs }\end{array}$} & Universitaire & Lycée & Collège & Primaire & Non scolarisés & Total \\
\hline & & & & & \multirow[t]{2}{*}{$39(38,61)$} & 101 \\
\hline & $4(3,96 \%)$ & $4(3,96 \%)$ & $32(31,68 \%)$ & $22(21,78 \%)$ & & $(100,00 \%)$ \\
\hline \multirow[t]{2}{*}{$\begin{array}{l}\text { Transmission des } \\
\text { connaissances } \\
\text { des PMTs }\end{array}$} & $\begin{array}{l}\text { Héritage } \\
\text { familial }\end{array}$ & $\begin{array}{l}\text { Initiation } \\
\text { traditionnelle }\end{array}$ & $\begin{array}{l}\text { Révélation } \\
\text { divine }\end{array}$ & $\begin{array}{l}\text { Utilisation } \\
\text { dans la } \\
\text { localité }\end{array}$ & - & \multirow{2}{*}{$\begin{array}{l}\text { Total } \\
101 \\
(100,00 \%) \\
\end{array}$} \\
\hline & $75(74,26 \%)$ & $6(5,94 \%)$ & $7(6,93 \%)$ & $13(12,87 \%)$ & & \\
\hline \multirow[t]{2}{*}{$\begin{array}{l}\text { Langues locales } \\
\text { des PMTs }\end{array}$} & Ewe & Guin & Kotokoli & Mina & Ouatchi & \multirow{2}{*}{$\begin{array}{l}\text { Total } \\
101 \\
(100,00 \%)\end{array}$} \\
\hline & $14(13,86 \%)$ & $12(11,88 \%)$ & $6(5,94 \%)$ & $7(6,93 \%)$ & $62(61,39 \%)$ & \\
\hline \multirow[t]{2}{*}{$\begin{array}{l}\text { Professions } \\
\text { principales des } \\
\text { PMTs }\end{array}$} & Agriculteur & Enseignant & PMTs & $\begin{array}{l}\text { Ménagère et } \\
\text { autres } \\
\text { professions }\end{array}$ & Revendeuse & \multirow{2}{*}{$\begin{array}{l}\text { Total } \\
101 \\
(100,00 \%)\end{array}$} \\
\hline & $46(45,54 \%)$ & $3(2,97 \%)$ & $33(32,67 \%)$ & $7(6,93 \%)$ & $12(11,88 \%)$ & \\
\hline
\end{tabular}

Tableau 2 : Liste des espèces végétales et leurs indices ethnobotaniques.

\begin{tabular}{lllll}
\hline No & Espèces & FC & CPR & ICF \\
\hline 1 & Acanthospermum hispidum DC & $0.84 \%$ & $1.00 \%$ & 0.04 \\
2 & Adansonia digitata L. & $1.26 \%$ & $1.00 \%$ & 0.06 \\
3 & Aframomum meleguetta (Roscoe) K.Schum. & $0.42 \%$ & $1.25 \%$ & 0.02 \\
4 & Allium cepa L. & $1.26 \%$ & $0.88 \%$ & 0.06 \\
5 & Allium sativum L. & $1.47 \%$ & $1.13 \%$ & 0.07 \\
6 & Aloe vera L. & $0.21 \%$ & $0.25 \%$ & 0.01 \\
7 & Alternanthera sessilis $($ L.) & $0.63 \%$ & $0.50 \%$ & 0.03 \\
8 & Amaranthus cruentus L. & $0.63 \%$ & $0.38 \%$ & 0.03 \\
9 & Amaranthus spinosus L. & $1.26 \%$ & $0.75 \%$ & 0.06 \\
10 & Amaranthus viridis L. & $1.05 \%$ & $0.63 \%$ & 0.05 \\
11 & Ananas comosus $($ L.) Merr. & $0.42 \%$ & $0.25 \%$ & 0.02 \\
12 & Anacardium occidentale L. & $0.21 \%$ & $0.13 \%$ & 0.01 \\
13 & Annona muricata L. & $0.84 \%$ & $1.00 \%$ & 0.04 \\
14 & Annona senegalensis Pers. & $0.21 \%$ & $0.25 \%$ & 0.01 \\
\hline
\end{tabular}




\begin{tabular}{|c|c|c|c|c|}
\hline 15 & Blighia sapida c. König & $0.21 \%$ & $0.13 \%$ & 0.01 \\
\hline 16 & Boerhavia diffusa $L$. & $1.68 \%$ & $1.38 \%$ & 0.08 \\
\hline 17 & Boerhavia erecta $L$. & $0.21 \%$ & $0.13 \%$ & 0.01 \\
\hline 18 & Brassica oleracea L. & $0.42 \%$ & $0.25 \%$ & 0.02 \\
\hline 19 & Byrsocarpus coccineus Schum. \& Thonn. & $1.26 \%$ & $1.50 \%$ & 0.06 \\
\hline 20 & Cajanus cajan $L$. & $0.21 \%$ & $0.25 \%$ & 0.01 \\
\hline 21 & Capsicum aппиит $L$. & $0.42 \%$ & $0.25 \%$ & 0.02 \\
\hline 22 & Capsicum frutescens & $0.42 \%$ & $0.25 \%$ & 0.02 \\
\hline 23 & Carica papaya $L$. & $1.26 \%$ & $0.88 \%$ & 0.06 \\
\hline 24 & Cassia occidentalis $L$. & $1.89 \%$ & $1.75 \%$ & 0.09 \\
\hline 25 & Ceasalpinia pulcherrima (L.) $S w$, & $0.21 \%$ & $0.50 \%$ & 0.01 \\
\hline 26 & Celosia argentea $L$. & $0.21 \%$ & $0.13 \%$ & 0.01 \\
\hline 27 & Centella asiatica $L$. & $0.21 \%$ & $0.38 \%$ & 0.01 \\
\hline 28 & Citrus aurantifolia Christm. Swingle. & $1.05 \%$ & $0.75 \%$ & 0.05 \\
\hline 29 & Citrus sinensis $L$. & $0.21 \%$ & $0.13 \%$ & 0.01 \\
\hline 30 & Cleome rutidosperma $D C$. & $0.63 \%$ & $0.38 \%$ & 0.03 \\
\hline 31 & Cleome gynandra $L$. & $0.84 \%$ & $1.00 \%$ & 0.04 \\
\hline 32 & Cocos nucifera $L$. & $0.84 \%$ & $0.50 \%$ & 0.04 \\
\hline 33 & Colocasia esculenta $L$. & $0.42 \%$ & $0.25 \%$ & 0.02 \\
\hline 34 & Corchorus aestuans $L$. & $2.94 \%$ & $2.63 \%$ & 0.14 \\
\hline 35 & Corchorus olitorius $L$. & $1.68 \%$ & $1.38 \%$ & 0.08 \\
\hline 36 & Corchorus tridens $L$. & $0.42 \%$ & $0.25 \%$ & 0.02 \\
\hline 37 & Crataeva adansonii $D C$. & $1.89 \%$ & $2.50 \%$ & 0.09 \\
\hline 38 & Croton zambesicus Müll. Arg. & $0.21 \%$ & $0.13 \%$ & 0.01 \\
\hline 39 & Cymbopogon citratus (DC.) Stapf. & $0.42 \%$ & $0.25 \%$ & 0.02 \\
\hline 40 & Dioscorea bulbifera $L$. & $0.21 \%$ & $0.13 \%$ & 0.01 \\
\hline 41 & Elaeis guineensis Jacq. & $0.63 \%$ & $0.38 \%$ & 0.03 \\
\hline 42 & Eucalyptus tereticornis Smith & $0.21 \%$ & $0.13 \%$ & 0.01 \\
\hline 43 & Euphorbia hirta L. & $0.21 \%$ & $0.13 \%$ & 0.01 \\
\hline 44 & Fagara zanthoxyloides Lam. & $2.73 \%$ & $2.63 \%$ & 0.13 \\
\hline 45 & Garcinia kola Heckel & $0.42 \%$ & $0.63 \%$ & 0.02 \\
\hline 46 & Gomphrena celosioides Mart & $0.21 \%$ & $0.13 \%$ & 0.01 \\
\hline 47 & Grewia barteri Burret & $1.89 \%$ & $2.00 \%$ & 0.09 \\
\hline 48 & Heliotropium indicum $L$. & $5.66 \%$ & $6.76 \%$ & 0.27 \\
\hline 49 & Hibiscus sabdariffa $L$. & $1.47 \%$ & $1.63 \%$ & 0.07 \\
\hline 50 & Ipomoea aquatica Forssk. & $2.10 \%$ & $1.75 \%$ & 0.10 \\
\hline
\end{tabular}




\begin{tabular}{|c|c|c|c|c|}
\hline 51 & Irvingia gabonensis Baill. & $0.21 \%$ & $0.38 \%$ & 0.01 \\
\hline 52 & Kalanchoe crenata Lam & $1.26 \%$ & $1.13 \%$ & 0.06 \\
\hline 53 & Lactuca taraxacifolia (Willd.) Schum. & $6.08 \%$ & $7.63 \%$ & 0.29 \\
\hline 54 & Laportea aestuans $L$. & $2.94 \%$ & $1.75 \%$ & 0.14 \\
\hline 55 & Mangnifera indica $L$. & $0.21 \%$ & $0.25 \%$ & 0.01 \\
\hline 56 & Manihot esculenta CRANTZ & $3.56 \%$ & $3.13 \%$ & 0.17 \\
\hline 57 & Moringa oleifera $L$. & $5.45 \%$ & $6.63 \%$ & 0.26 \\
\hline 58 & Ocimum canum Sims & $0.63 \%$ & $0.75 \%$ & 0.03 \\
\hline 59 & Ocimum gratissimum $L$. & $10.48 \%$ & $11.76 \%$ & 0.50 \\
\hline 60 & Parkia biglobosa Benth. & $0.42 \%$ & $0.38 \%$ & 0.02 \\
\hline 61 & Paullinia pinnata $L$. & $0.21 \%$ & $0.13 \%$ & 0.01 \\
\hline 62 & Persea americana Mill. & $0.42 \%$ & $0.25 \%$ & 0.02 \\
\hline 63 & Phyllanthus amarus Schum. & $1.05 \%$ & $1.00 \%$ & 0.05 \\
\hline 64 & Physalis angulata $L$. & $0.42 \%$ & $0.38 \%$ & 0.02 \\
\hline 65 & Portulaca oleracea $L$. & $0.42 \%$ & $1.00 \%$ & 0.02 \\
\hline 66 & Psidium guajava $L$. & $0.42 \%$ & $0.38 \%$ & 0.02 \\
\hline 67 & Pterocarpus santalinoides DC. & $0.21 \%$ & $0.38 \%$ & 0.01 \\
\hline 68 & Raphia hookeri Mann \& Wendl. & $0.21 \%$ & $0.13 \%$ & 0.01 \\
\hline 69 & Solanum aethiopicum $L$. & $0.42 \%$ & $0.38 \%$ & 0.02 \\
\hline 70 & Solanum americanum $L$. & $1.47 \%$ & $1.13 \%$ & 0.07 \\
\hline 71 & Solanum lycopersicum $L$. & $0.21 \%$ & $0.13 \%$ & 0.01 \\
\hline 72 & Solanum macrocarpum $L$. & $0.84 \%$ & $0.75 \%$ & 0.04 \\
\hline 73 & Sorghum caudatum (Hack.) Stapf & $0.21 \%$ & $0.13 \%$ & 0.01 \\
\hline 74 & Spondias monbin $L$. & $1.26 \%$ & $1.00 \%$ & 0.06 \\
\hline 75 & Syzygium aromaticum $L$. & $0.63 \%$ & $0.63 \%$ & 0.03 \\
\hline 76 & Talinum triangulare (Jacq.) Willd & $0.84 \%$ & $0.75 \%$ & 0.04 \\
\hline 77 & Tamarindus indica $L$. & $0.21 \%$ & $0.25 \%$ & 0.01 \\
\hline 78 & Terminalia glaucescens Planch. ex Benth. & $0.21 \%$ & $0.13 \%$ & 0.01 \\
\hline 79 & Tetrapleura tetraptera (Schumach. \& Thonn.) & $0.84 \%$ & $0.88 \%$ & 0.04 \\
\hline 80 & Tridax procumbens $L$. & $1.68 \%$ & $1.38 \%$ & 0.08 \\
\hline 81 & Uvaria chamae $L$. & $0.42 \%$ & $0.50 \%$ & 0.02 \\
\hline 82 & Vernonia amygdalina Delile & $6.71 \%$ & $7.76 \%$ & 0.32 \\
\hline 83 & Vitex doniana Sweet & $1.89 \%$ & $1.63 \%$ & 0.09 \\
\hline 84 & Xylopia aethiopica A. Rich. & $0.21 \%$ & $0.50 \%$ & 0.01 \\
\hline 85 & Zea maïs $L$. & $0.21 \%$ & $0.13 \%$ & 0.01 \\
\hline 86 & Zingiber officinale Roscoe & $0.63 \%$ & $1.00 \%$ & 0.03 \\
\hline
\end{tabular}




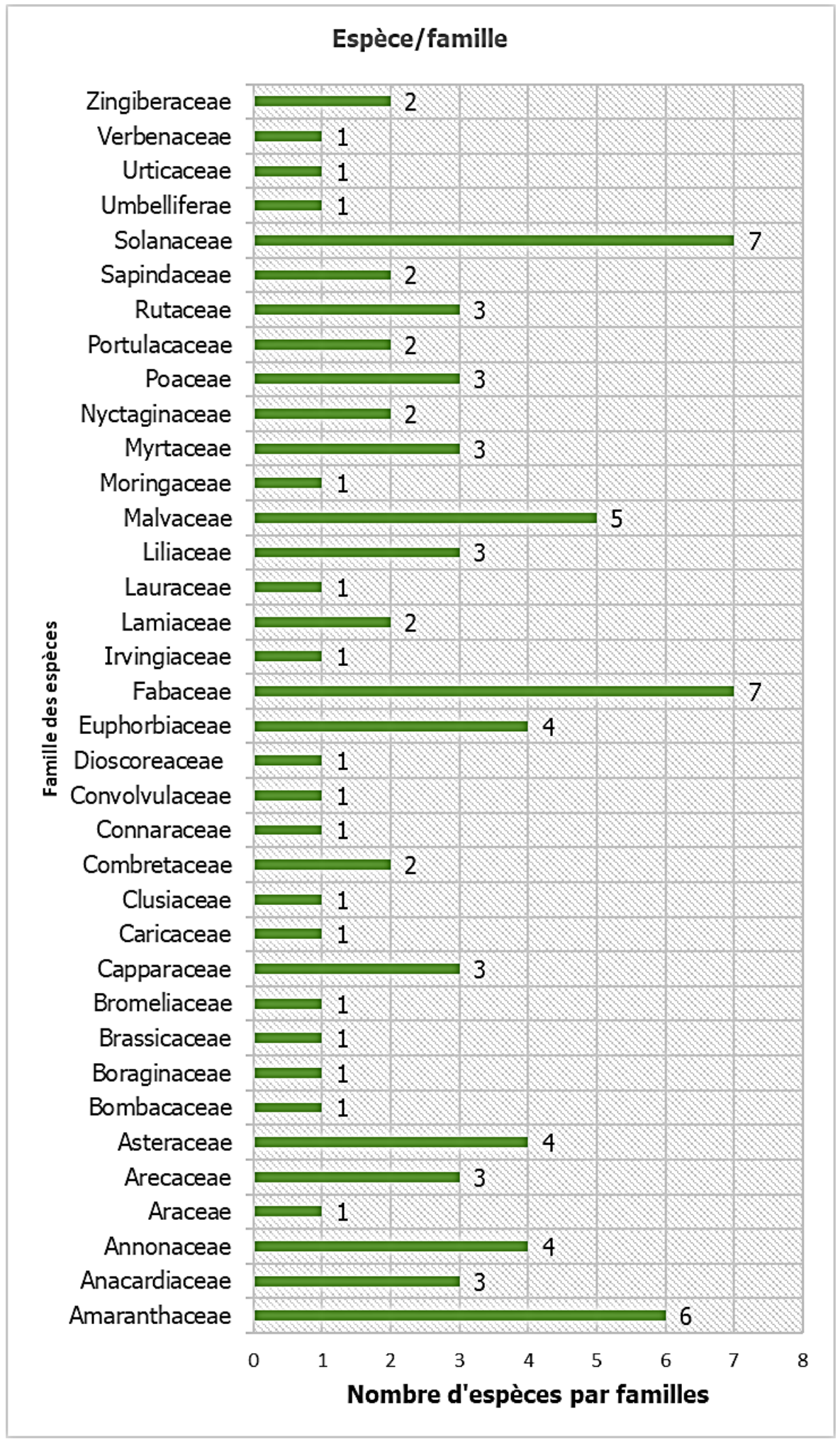

Figure 2 : Diversité botanique des plantes aux potentiels nutritionnel et thérapeutique de la région Maritime du Togo. 


\section{Pourcentage des Légumes et Légumes-feuilles par rapport aux autres espèces}

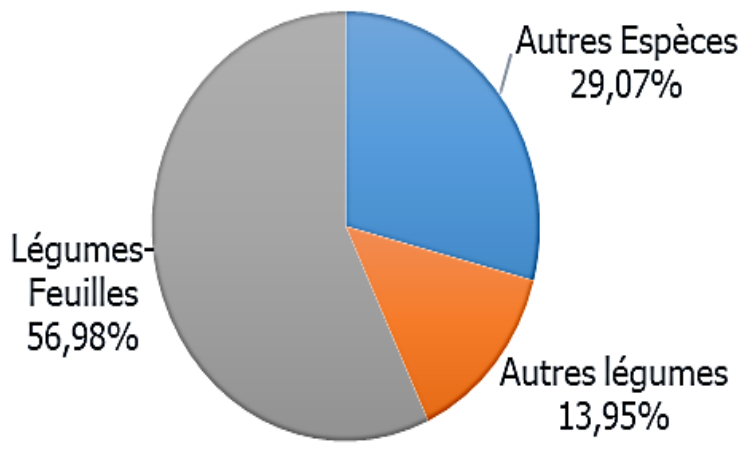

$\square$ Autres Espèces $\quad$ Autres légumes $\quad$ Légumes-Feuilles

Figure 3 : Proportion des légumes et légumes-feuilles par rapport aux autres espèces.

Tableau 3 : Parties des plantes, formes galéniques et mode d'administration utilisés.

\begin{tabular}{cccccc}
\hline Parties utilisées & \% & Formes galéniques & \% & Mode d'administration & \% \\
\hline Autres parties & $1,38 \%$ & Alcoolature & $1,88 \%$ & Auditive & $0,38 \%$ \\
Bulbes & $2,00 \%$ & Autres formes & $0,63 \%$ & Bain & $1,00 \%$ \\
Ecorces & $3,13 \%$ & Pâte & $1,13 \%$ & Cutanée & $3,00 \%$ \\
Feuilles & $77,85 \%$ & Décocté & $14,14 \%$ & Gargarisme & $0,38 \%$ \\
Fruits & $5,63 \%$ & Infusé & $6,13 \%$ & Nasale & $2,38 \%$ \\
Graines & $3,25 \%$ & Jus & $14,52 \%$ & Oculaire & $1,38 \%$ \\
Plante entière & $0,50 \%$ & Macéré & $0,88 \%$ & Olfactive & $0,13 \%$ \\
Racines & $4,26 \%$ & Matières crues & $6,63 \%$ & Orale & $90,74 \%$ \\
Rhizomes & $1,00 \%$ & Poudre & $2,88 \%$ & Oto-Rhino-Laryngologique & $0,38 \%$ \\
Tige & $0,50 \%$ & Sauce & $51,19 \%$ & Rectale & $0,25 \%$ \\
Tubercules & $0,50 \%$ & - & - & - & - \\
\hline
\end{tabular}


Tableau 4 : Fréquence des catégories de maladies traitées.

\begin{tabular}{lll}
\hline Catégories d'affections & $\begin{array}{l}\text { Nombre d'indications thérapeutiques } \\
\text { ou affections répertoriées }\end{array}$ & Fréquence \\
\hline Affections cutanées & 30 & $3,75 \%$ \\
\hline Autres affections & 26 & $3,25 \%$ \\
\hline Affections asthéniques & 59 & $7,38 \%$ \\
\hline Affections hématologiques & 39 & $4,88 \%$ \\
\hline Affections oto-rhino-laryngologiques & 26 & $3,25 \%$ \\
\hline Affections cardiovasculaires & 108 & $13,52 \%$ \\
\hline Affections ophtalmologiques & 10 & $1,25 \%$ \\
\hline Affections du tube digestif & 350 & $43,80 \%$ \\
\hline Affections endocrino-métaboliques & 15 & $1,88 \%$ \\
\hline Affections gynécologiques et obstétricales & 6 & $0,75 \%$ \\
\hline Affections mystiques & 16 & $2,00 \%$ \\
\hline Affections neurologiques & 4 & $0,50 \%$ \\
\hline Affections uro-génitales & 10 & $1,25 \%$ \\
\hline Affections bucco-dentaires & 13 & $1,63 \%$ \\
\hline Affections rhumatologiques & 6 & $0,75 \%$ \\
\hline Affections infectieuses & 49 & $6,13 \%$ \\
\hline Affections respiratoires & 32 & $4,01 \%$ \\
\hline & & \\
\hline
\end{tabular}

\section{DISCUSSION}

La présente étude avait pour objectif de recenser les plantes aux potentiels nutritionnel et thérapeutique utilisées dans la région Maritime du Togo. Auparavant, des études ont été menées dans cette région concernant: le paludisme (Koudouvo et al., 2011 ; Agbodeka et al., 2016), le diabète (Gbekley et al., 2015), les atteintes pathologiques du foie (Kpodar et al., 2016), les maladies cardiovasculaires (Gbekley et al., 2018). Cette étude qui concerne les plantes aux potentiels nutritionnel et thérapeutique répond à l'inquiétude grandissante du double fardeau nutritionnel qui menace la santé au Togo. Il s'agit d'une première étude du genre réalisée dans la région Maritime du Togo. L'enquête ethnobotanique a été réalisée auprès de 101 PMTs qui étaient majoritairement des femmes adultes. Ce profil des PMTs de la région Maritime du Togo est contraire à celui observé dans la plupart des études réalisées dans cette région concluant que la pratique de la médecine traditionnelle est l'apanage des hommes d'âge mûr (Gbekley et al., 2015). Une analyse contextuelle de l'enquête nous permet de comprendre que les plantes aux potentiels nutritionnels même si elles sont aussi utilisées en thérapies, interviennent plus dans les ménages et surtout dans l'alimentation dont les femmes sont les vraies détentrices des savoirs et pratiques culinaires. En effet, les plantes alimentaires sont plus connues et utilisées par les femmes dans la tradition africaine ; ce qui explique le fait qu'elles sont majoritaires dans l'effectif. Ces plantes qui font objet des savoirs et pratiques culinaires sont connues par les femmes en premier et transmises de mère à 
fille. Cette transmission est observée aussi au marché de la bonne femme qui vend, à la domestique qui l'accompagne dans la vente des parties des plantes et/ou à l'acheteuse. La prédominance de femmes s'explique en partie par le fait que les enquêtes ont été menées aussi dans les ménages où les femmes sont plus représentées. Diverses études en Afrique subsaharienne ont démontré le rôle prépondérant des femmes dans la gestion des plantes légumières. Selon nombre d'auteurs, en plus du mode de prélèvement, la valorisation et la gestion durable de ces ressources tiennent compte des réalités socioculturelles et du partage des tâches dans les communautés rurales. En effet, les femmes, aidées parfois par les enfants, ont en charge les activités de cueillette, de conditionnement et de commercialisation des plantes légumières (Nesamvuni et al., 2001; Gockowski et al., 2003). Selon ces études, la connaissance d'une recette en médecine traditionnelle est avant tout un secret de famille qui est transmis de génération en génération par le biais des coutumes et de la tradition orale. Il est donc nécessaire d'avoir un âge mature et de se faire une certaine confiance pour avoir accès aux connaissances de cette médecine et c'est probablement la principale raison pour laquelle ce métier est pratiqué par des personnes âgées.

L'étude a montré une bonne diversité des plantes aux potentiels nutritionnel et thérapeutique dans la région Maritime du Togo avec 86 espèces végétales appartenant à 36 familles recensées. Il ressort alors de cette étude que les espèces les plus utilisées par les PMTs de la région Maritime pour les 799 recettes inventoriées sont $O$. gratissimum $(94$ recettes), $V$. amygdalina (62 recettes), $L$. taraxacifolia (61 espèces), $H$. indicum (54 recettes), $M$. oleifera (53 recettes), $M$. esculenta (25 recettes), $C$. aestuans et $F$. zanthoxyloides (21 recettes), C. adansonii (20 recettes). L'importance de ces plantes a été décelée par la résultante des indices ethnobotaniques et de l'évidence ethnonutritionnelle et thérapeutique. L'indice de consensus $(0,5)$ obtenu pour $O$. gratissimum, indique qu'il y a un accord élevé autour de son usage alimentaire et thérapeutique. Cela peut s'expliquer par la proximité culturelle des enquêtés. Par ailleurs, les espèces légumières inventoriées dans cette étude viennent corroborer la diversité relativement élevée de légumes de cueillette au Togo (Batawila et al., 2007). En fait cette diversité des plantes aux potentiels nutritionnel et thérapeutique (plantes légumières et surtout de cueillette) est liée aux différents lieux de prélèvement que sont les jardins familiaux, les formations végétales environnantes (savanes, forêts, buissons, fourrés), les jachères, les champs (Batawila et al., 2007).

Les récoltes des plantes aux potentiels nutritionnel et thérapeutique sont faites en toutes heures et à n'importe quel moment de l'année selon la disponibilité des espèces. D'autres études ont révélé la même conclusion et s'accordent sur la période de disponibilité des légumes de cueillette récoltés en saison pluvieuse qui sont séchés, réduits en poudre et conservés pour couvrir la saison sèche ou les périodes de soudure (Shiundu, 2002 ; Batawila et al., 2007 ; Gbekley et al., 2015).

Les familles les plus représentées dans la région Maritime du Togo ont été les Solanaceae $(8,14 \%)$, les Fabaceae $(8,14 \%)$, les Amaranthaceae $(6,98 \%)$ et les Malvaceae $(5,81 \%)$. Ces résultats ne reflètent pas la tendance dans des études similaires au Bénin où les familles d'espèces botaniques les plus représentées sont celles des Amaranthaceae $(23,53 \%)$ et des Lamiaceae $(17,65 \%)$ (Dansi et al., 2008 ; Agbankpé et al., 2014) de même qu'au Nigéria où les familles les plus représentées sont celles des Asteraceae et des Cucurbitaceae (Omara-Achong et al., 2012). Cependant, ces résultats présentent quelques similitudes avec d'autres études ethnobotaniques qui ont révélé de pareilles diversités de plantes.

Selon l'étude réalisée au Togo (Batawila et al., 2007), au total, 105 espèces 
dont un ou plusieurs organes sont utilisés comme légumes, ont été recensées. Elles ont été rangées dans 82 genres et 45 familles dont les Fabaceae, les Malvaceae, les Moraceae et les Asteraceae ont été les plus nombreuses. Les espèces les plus citées ont été Adansonia digitata, F. zanthoxyloides, O. gratissimum, Talinum triangulare, V. amygdalina et Vitex doniana. Les organes les plus utilisés ont été les feuilles (67\%) et les fruits (18\%) ; suivis par les fleurs $(6 \%)$ et les racines $(6 \%)$.

L'étude réalisée au Bénin à l'aide d'enquêtes basées sur la méthode d'interview semi-structurée a permis d'identifier les légumes-feuilles à propriétés anti-diarrhéiques, consommés à Cotonou et Abomey-Calavi (Sud-Bénin). Elle a recensé 27 espèces de légumes-feuilles regroupées en 23 genres et appartenant à 17 familles botaniques. Les familles les plus représentées ont été les Amaranthaceae et les Lamiaceae. Les espèces les plus consommées pour le traitement des infections diarrhéiques ont été : O. gratissimum $(29,05 \%), \quad V$. amygdalina $(16,80 \%), \quad C$. adansonii $(13,49 \%)$ et Sesamum radiatum L. $(11,41 \%)$. Ces légumes-feuilles sont préparés à l'état frais ou sec (poudres) puis consommés comme légumes d'accompagnement (sauce) ou utilisés comme tisane.

$\mathrm{Au}$ Cameroun, l'étude utilisant des interviews directes et semi-structurées a permis d'identifier 84 espèces réparties en 78 genres et 50 familles dont les plus représentées ont été les Asteraceae ( 9 espèces), Fabaceae (6 espèces) et Lamiaceae (4 espèces) (Mpondo Mpondo et al., 2012).

Les affections les plus traitées par les plantes alimentaires sont en majorité les affections du tube digestif $(43,80 \%)$. Cette tendance est similaire aux résultats d'une étude dans le sud-ouest Algérien où le taux le plus élevé d'espèces de plantes médicinales $(43,47 \%)$, intervenaient dans le traitement des maladies digestives (Kadri et al., 2018). Au Maroc, ce sont les affections du tube digestif qui sont les plus traitées avec les plantes médicinales, avec un pourcentage de $34,6 \%$ (El
Hafian et al., 2014) et 37,78\% (Rhattas et al., 2016). Au Cameroun des études similaires ont classé également les affections du tube digestif en tête des affections traitées par les plantes (Betti, 2002). Toutefois, dans d'autres études toujours au Cameroun, les maladies infectieuses prévalent (66\%) comme les plus traitées (Ladoh-Yemeda et al., 2016).

Cette étude valorise à double titre les plantes : d'une part, leurs potentiels nutritionnels car d'une manière générale, l'utilisation de ces plantes légumières est liée à leur richesse en substances nutritives (protéines, éléments minéraux) (Gockowski et al. 2003; Batawila et al., 2007); d'autre part, leurs potentiels thérapeutiques car les plantes alimentaires riches en minéraux, vitamines et métabolites secondaires possèdent des propriétés médicinales (Gbekley et al., 2015).

\section{Conclusion}

Il ressort de cette étude que la région Maritime du Togo dispose d'une biodiversité floristique intéressante en matière de plantes d'intérêts thérapeutique et nutritionnel. Elle a souligné le rôle essentiel des herboristes, des phytothérapeutes et des ménages dans le traitement des affections à partir des plantes alimentaires au Togo. De plus, les PMTs de la région Maritime présentent une bonne diversité dans l'utilisation des espèces végétales. Les plantes ainsi répertoriées constituent un panel qui peut servir de point de départ pour les criblages biologiques au laboratoire. En ce sens, ces études permettront d'une part, d'évaluer le potentiel nutritionnel et thérapeutique et d'autre part, d'isoler et d'identifier des principes actifs qui pourraient donner lieu à des alicaments pour la promotion de la santé de la population. Face à la problématique du double fardeau nutritionnel, cette étude n'est qu'une étape d'un programme qui se poursuivra par la valorisation effective du potentiel nutritionnel et thérapeutique des plantes alimentaires et légumières à travers certains produits à valeurs ajoutées, tels que la « moutarde traditionnelle » de graines de néré 
(Parkia biglobosa) et les additifs alimentaires naturels. Ainsi, on pourra atteindre un des objectifs de l'Organisation Ouest Africaine de la Santé (OOAS) qui se veut de valoriser tous les potentiels de nos savoirs ethno-médicinaux pour une santé publique efficace.

\section{CONFLIT D'INTERETS}

Les auteurs ne déclarent aucun conflit d'intérêt par rapport à cet article.

\section{CONTRIBUTIONS DES AUTEURS}

ES, GEH, AA, TT, OE, KSD et KK : Initiation et élaboration du protocole, ES et GEH : Collecte des données, KK : Identification des plantes, ES et GEH : Traitement des données, ES, GEH, MM, AA, TT : Rédaction du manuscrit.

\section{REMERCIEMENTS}

Les auteurs remercient tous les phytothérapeutes, les herboristes et les ménages de la région maritime du Togo de leur franche collaboration qui a rendu possible cette étude.

\section{REFERENCES}

Agban A, Atchou K, Effoe S, Karou SD, Batawila K, Tchacondo T, de Souza C, Gbeassor M, Akpagana K. 2013. Evaluation du potentiel antimicrobien de Piliostigma thonningii (Schumach.) Milne Redh. (Fabaceae). Revue Africaine de Santé et de Protections Animales, 11(2): 101-106.

Agbankpé J, Dougnon TV, Bankole HS, Yèhouénou B, Yedomonhan $\mathrm{H}$, Legonou M, Dougnon TJ. 2014. Etude ethnobotanique des légumes feuilles thérapeutiques utilisés dans le traitement des diarrhées au sud-Bénin (Afrique de l'Ouest). Int. J. Biol. Chem. Sci., 8(4): 1784-1795. DOI: 10.4314/ijbcs.v8i4.36

Agbodeka K, Gbekley HE, Karou SD, Anani K, Agbonon A, Tchacondo T, Batawila K, Simpore J, Gbeassor M. 2016. Ethnobotanical study of medicinal plants used for the treatment of malaria in the plateau region, Togo. Pharmacognosy research, 8(1): S12-S18. DOI: 10.4103/0974-8490.178646.

Akpavi S. 2010. Plantes alimentaires mineures ou menacées de disparition au Togo: diversité, ethnobotanique et valeurs. Acta Botanica Gallica, 157(2): 379-383. DOI: 10.1080/12538078.2010.10516215

Akpavi S, Woegan A, Dourma M, Tozo K, Batawila K, Wala K, Gbogbo K, Kanda M, Kossi-Titrikou K, de Foucault B. 2011. Que sont devenues les plantes autrefois consommées par les divers groupes ethnoculturels du Togo? Agronomie Africaine, 23(2): 147-160.

Batawila K, Akpavi S, Wala K, Kanda M, Vodouhe R, Akpagana K. 2007. Diversité et gestion des légumes de cueillette au Togo. African Journal of Food, Agriculture, Nutrition And Development, 7(3-4).

Benem B, Sanou-Nana P. 2009. Plantes médicinales utilisées pour le soin des enfants dans la ville de Ouagadougou, Burkina Faso, Afrique de l'Ouest. Le Flamboyant, 65: 9-12.

Benkhnigue O, Zidane L, Fadli M, Elyacoubi H, Rochdi A, Douira A. 2010. Etude ethnobotanique des plantes médicinales dans la région de Mechraâ Bel Ksiri (Région du Gharb du Maroc). Acta Botanica Barcinonensia, 53: 191-216.

Betti JL. 2002. Medicinal plants sold in Yaoundé markets, Cameroon. African Study Monographs, 23(2): 47-64. DOI: 10.14989/68215.

Betti JL, Mebere-Yemefa'a SR. 2011. Contribution à la connaissance des produits forestiers non ligneux du parc national de Kalamaloué, Extrême-Nord Cameroun : les plantes alimentaires. Int. J. Biol. Chem. Sci., 5(1): 291-303. DOI: 10.4314/ijbcs.v5i1.68105

Betti JL, Ngankoué CM, Dibong SD, Singa AE. 2016. Etude ethnobotanique des plantes alimentaires spontanées vendues 
dans les marchés de Yaoundé, Cameroun.

Int. J. Biol. Chem. Sci., 10(4): 1678-1693.

DOI:

http://dx.doi.org/10.4314/ijbcs.v10i4.19

Dansi A, Adjatin A, Vodouhè R, Adéoti K, Adoukonou-Sagbadja $\mathrm{H}$, Faladé V, Yédomonhan $\mathrm{H}$, Akoègninou $\mathrm{A}$, Akpagana K. 2008. Biodiversité des Légumes Feuilles Traditionnels Consommés au Bénin. Bibliothèque nationale: Bénin.

Dassou H, Ogni C, Yédomonhan H, Adomou A, Tossou M, Dougnon J, Akoègninou A. 2014. Diversité, usages vétérinaires et vulnérabilité des plantes médicinales au Nord-Bénin. Int. J. Biol. Chem. Sci., 8(1): 189-210. DOI: 10.4314/ijbcs.v8i1.18

Direction de la Statistique et de la Comptabilité

Nationale. 2010. RGPH, Résultats définitifs du Recensement Général de la Population du 06 au 21 novembre 2010. DGSCN/PNUD/UNICEF, Lomé, 252p.

El Hafian M, Benlamdini N, Elyacoubi H, Zidane L, Rochdi A. 2014. Étude floristique et ethnobotanique des plantes médicinales utilisées au niveau de la préfecture d'Agadir-Ida-Outanane, Maroc. Journal of Applied Biosciences, 81: 7198-7213. DOI: 10.4314/jab.v81i1.8.

Gayibor NL. 2006. Histoire des Togolais de 1884 à 1960 (2 tomes). Presses de l'Université de Lomé.

Gbekley HE, Karou DS, Katawa G, Tchacondo T, Batawila K, Ameyapoh Y. 2018. Ethnobotanical survey of medicinal plants used in the management of arterial hypertension in the Maritime Region of Togo. African Journal of Traditional, Complementary and Alternative Medicines, 15(1): 85-97. DOI: 10.21010/ajtcam.v15i1.9.

Gbekley HE, Karou SD, Gnoula C, Agbodeka K, Anani K, Tchacondo T, Agbonon A, Batawila K, Simpore J. 2015. Étude ethnobotanique des plantes utilisées dans le traitement du diabète dans la médecine traditionnelle de la région Maritime du Togo. Pan African Medical Journal, 20: 437.

DOI: 10.11604/pamj.2015.20.437.5660.

Gockowski J, Mbazo'o J, Mbah G, Moulende TF. 2003. African traditional leafy vegetables and urban and peri-urban poor. Food Policy, 28(3): 221-235. DOI: https://doi.org/10.1016/S03069192(03)00029-0.

Houéhanou D, Assogbadjo A, Chadare F, Zanvo S, Sinsin B. 2016. Approches méthodologiques synthétisées des études d'ethnobotanique quantitative en milieu tropical. Annales des sciences agronomiques, 20: 187-205.

Ilumbe GB, Van Damme P, Lukoki FL, Joiris V, Visser M, Lejoly J. 2014. Contribution à l'étude des plantes médicinales dans le traitement des hémorroïdes par les pygmées Twa et leur voisin Oto de Bikoro, en RDC. Congo Sci., 2(1): 46-54. Jiofack T, Fokunang C, Guedje N, Kemeuze V, Fongnzossie E, Nkongmeneck BA, Mapongmetsem PM, Tsabang N. 2010. Ethnobotanical uses of medicinal plants of two ethnoecological regions of Cameroon. International Journal of Medicine and Medical Sciences, 2(3): 6079.

Kadri Y, Moussaoui A, Benmebarek A. 2018. Étude ethnobotanique de quelques plantes médicinales dans une région hyper aride du Sud-ouest Algérien « Cas du Touat dans la wilaya d'Adrar ». Journal of Animal \& Plant Sciences, 36(2): 58445857.

Kokou K, Adjossou K, Hamberger K. 2005. Les forêts sacrées de l'aire Ouatchi au sud-est du Togo et les contraintes actuelles des modes de gestion locale des ressources forestières. VertigO, 6 (3): DOI: $10.4000 /$ vertigo. 2456

Koudouvo K, Karou DS, Kokou K, Essien K, Aklikokou K, Glitho IA, Simporé J, Sanogo R, de Sousa C, Gbeassor M. 2011. An ethnobotanical study of 
antimalarial plants in Togo Maritime Region. Journal of Ethnopharmacology, 134(1): 183-190.

DOI: https://doi.org/10.1016/j.jep.2010.12.011 Kpodar MS, Karou SD, Katawa G, Anani K, Gbekley HE, Adjrah Y, Tchacondo T, Batawila K, Simpore J. 2016. An ethnobotanical study of plants used to treat liver diseases in the Maritime region of Togo. Journal of Ethnopharmacology, 181: 263-273. DOI: https://doi.org/10.1016/j.jep.2015.12.051 Ladoh-Yemeda CF, Vandi T, Dibong SD, Mpondo EM, Wansi JD, Betti JL, Choula F, Ndongo D, Eyango MT. 2016. Étude ethnobotanique des plantes médicinales commercialisées dans les marchés de la ville de Douala, Cameroun. Journal of Applied Biosciences, 99(1): 9450-9466. DOI: 10.4314/jab.v99i1.11.

Mpondo Mpondo E, Dibong SD, Ladoh Yemeda CF, Priso RJ, Ngoye A. 2012. Les plantes à phénols utilisées par les populations de la ville de Douala. Journal of Animal and Plant Sciences, 15(1): 2083-2098.
Nesamvuni C, Potgieter MJ, Steyn NP. 2001. Nutritional value of wild, leafy plants consumed by the Vhavenda. South African Journal of Science, 97(1): 51-54.

Omara-Achong TE, Edwin-Wosu NL, Edu EA, Nkang AE. 2012. Survey of indigenous vegetables species in parts of Ogoja and Cala Bar, Cross River State, Nigeria. Euro. J. Exp. Bio., 2(4): 1289-1301.

OMS. 2012. Statistiques sanitaires mondiales. OMS, 198p.

Rhattas M, Douira A, Zidane L. 2016. Étude ethnobotanique des plantes médicinales dans le Parc National de Talassemtane (Rif occidental du Maroc). Journal of Applied Biosciences, 97: 9187-9211. DOI: 10.4314/jab.v97i1.5.

Salhi S, Fadli M, Zidane L, Douira A. 2010. Etudes floristique et ethnobotanique des plantes médicinales de la ville de Kénitra (Maroc). Lazaroa, 31: 133-146. DOI:10.5209/rev_LAZA.2010.v31.9

Shiundu KM. 2002. Role of African leafy vegetables (ALVs) in alleviating food and nutrition insecurity in Africa. Afr. J. Food Nutr. Sci., 2(2): 97-99. 